The author is indebted to the University of California (Berkeley) Computer ('enter for its cooperation.

University of California

Berkeley, California

1. Richard Chandler \& Robert Spira, Enumeration of 2-complexes. (To appear.)

2. D. KöNIG, Theorie der endlichen und unendlichen Graphen, Chelsea, New York, 1950, p. 52. MR 12, 195 .

\title{
Generation of Triangulations of the Sphere
}

\section{By Robert Bowen and Stephen Fisk}

It is easily seen that there is only one triangulation of the sphere with four vertices and one with five. This paper concerns an algorithm for finding all (nonisomorphic) triangulations of the 2 -sphere with $N$ vertices from those with $N-1$. "Triangulation" shall always refer to a triangulation of the 2 -sphere. First we develop a method for generating all triangulations with $N$ vertices which may yield several triangulations of the same isomorphism type, and then we describe an isomorphism routine for eliminating these duplications.

Let $T$ be a triangulation with $N \geqq 5$ vertices, $E$ edges, and $F$ faces. Let $X_{k}$ denote the number of vertices of $T$ of valency $k$. Then $3 F=2 E$ as each face is a triangle and each edge is on two faces, and $2 E=\sum k X_{k}$ as each edge is incident to two vertices. Hence $6 F-6 E=-2 E=-\sum k X_{k}$ and by Euler's formula we have

$$
12=6 N+6 F-6 E=6 N-\sum k X_{k}=\sum X_{k}(6-k) .
$$

Since $\sum X_{k}(6-k)$ is positive, $T$ must have a vertex of valency less than six. Because every edge of $T$ must lie on two distinct triangular faces, each vertex must have valency greater than two. Letting $Q$ be a vertex of minimal valency, $Q$ must have valency three, four, or five.

Case 1. Suppose $Q$ has valency three. Then, about $Q, T$ has the form shown in Fig. 1. Removing $Q$ and the edges $Q P_{k}$, we obtain a triangulation $T^{\prime}$ with $N-1$ vertices. Thus we obtain $T$ if we add the point $Q$ to the center of the face $P_{1} P_{2} P_{3}$ and add the edges $Q P_{k}(k=1,2,3)$.

Case 2. Suppose $Q$ has valency four. Then, about $Q, T$ has the form shown in Fig. 2. By the Jordan curve theorem either $P_{1}$ is not adjacent to $P_{3}$ or $P_{2}$ is not adjacent to $P_{4}$; say $P_{1}$ is not adjacent to $P_{3}$. Then, removing $Q$ and edges $Q P_{k}$ $(1 \leqq k \leqq 4)$ and adding edge $P_{1} P_{3}$ inside the quadrilateral $P_{1} P_{2} P_{3} P_{4}$, we obtain a triangulation $T^{\prime}$ with $N-1$ vertices. The slight complication here is needed to insure that $T^{\prime}$ is a triangulation; for if $P_{1}$ were adjacent to $P_{3}$ in $T$, then $T^{\prime}$ would have multiple edges and would not be a triangulation. We now obtain $T$ from $T^{\prime}$ by reversing the process.

Case 3. Assume $Q$ has valency five. We claim some $P_{k}$ is adjacent to no $P_{i}$ other than the two shown (Fig. 3 ). Otherwise $P_{1}$ would be adjacent to $P_{3}$ or $P_{4}$, say $P_{3}$. Then by the Jordan curve Theorem, $P_{2}$ could be adjacent to neither $P_{4}$ nor

Received October 25, 1965. 


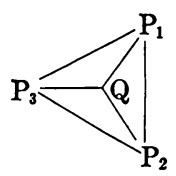

Figure 1

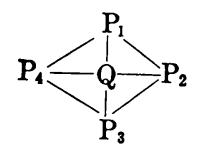

Figure 2<smiles>C12P3P4P1P3P24</smiles>

Figure 3

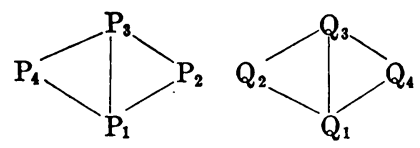

Figure 4

$P_{5}$. Hence we may assume (perhaps renumbering the $P^{\prime} \mathrm{s}$ ) that $P_{1}$ is not adjacent to $P_{3}$ or $P_{4}$. Now removing $Q$ and edges $Q P_{k}$ and adding edges $P_{1} P_{3}$ and $P_{1} P_{4}$ we obtain a triangulation $T^{\prime}$. Reversing the process we again obtain $T$.

We thus have three operations which when applied in all possible ways to all triangulations of $N-1$ vertices $(N \geqq 5$ ) will yield all triangulations of $N$ vertices.

Now suppose $T_{1}$ and $T_{2}$ are two triangulations of $N$ vertices. Ordering the faces of $T_{k}$ according to the largest valency of a vertex of the face, then the second largest, and finally the third valency, let $M_{k}$ be the set of maximal faces of $T_{k}$. Let $P_{1} P_{2} P_{3}$ be in $M_{2}$. Then an isomorphism $F$ of $T_{1}$ onto $T_{2}$ must have $F\left(Q_{i}\right)=P_{i}$ for some $Q_{1} Q_{2} Q_{3}$ in $M_{1}$. Suppose $F^{*}$ maps $Q_{i}$ into $P_{i}$ and we wish to extend $F^{*}$ to a map of the whole vertex set of $T_{1}$ which induces an isomorphism of $T_{1}$ onto $T_{2}$. Moving clockwise from $Q_{3}$, let $Q_{4}$ be the next point adjacent to $Q_{1}$. As all faces of $T_{1}$ are triangles, $Q_{3}$ is adjacent to $Q_{4}$. Let $P_{4}$ be the vertex other than $P_{2}$ of $T_{2}$ which lies on a face with $P_{1}$ and $P_{3}$. If $F$ is an isomorphism extending $F^{*}$, then as

$$
F\left(Q_{4}\right) \neq F\left(Q_{2}\right)=P_{2}
$$

and $F\left(Q_{4}\right)$ is adjacent to $F\left(Q_{1}\right)=P_{1}$ and $F\left(Q_{3}\right)=P_{3}$ we must have $F\left(Q_{4}\right)=P_{4}$. Continuing in this manner we see that $F$ is determined at every vertex adjacent to $Q_{1}$. Each of these vertices then lies on a face at which $F$ is determined; hence repeating the argument at these vertices, $F$ is determined at all vertices at a (graph theoretical) distance two from $Q_{1}$. By induction this method determines the isomorphism $F$ extending $F^{*}$ if it exists. Applying this algorithm to all possible $F^{* \text { 's }}$ and testing each $F$ for isomorphism, we decide whether $T_{1}$ and $T_{2}$ are isomorphic.

In applying the generation algorithm to $T^{\prime}$ with $N-1$ vertices, we do not use 
the third operation described above if its yields a triangulation with a valency three or four, or the second if it gives a triangulation with a valency three; for such triangulations will be obtained by applying earlier operations to another triangulation of $N-1$ vertices. From formula (1) we see that if $T$ has no valency three or four, then it has at least twelve vertices of valency five. A triangulation with twelve vertices of valency five is a regular polyhedron; there is only one such, the wellknown icosahedron. Thus in obtaining all triangulations with $N \leqq 12$ vertices one need never apply the third operation, which in practice is by far the most time consuming. The generation of all such triangulations was carried out on the IBM 7094 in approximately $1 \frac{1}{2}$ hours of computing time. As a check, the computation was carried out for $N \leqq 11$ with a general graph isomorphism routine (see [1] for a brief description of this routine). D. W. Grace [2] generated all trihedral polyhedra (the dual of triangulations) with 11 or fewer faces and our numbers check with his to that point. In the table below $L(N)$ is the number of triangulations and $M(N)$ the number with no valency three with $N$ vertices.

$\begin{array}{rcccrc}N & L(N) & M(N) & N & L(N) & M(N) \\ 6 & 2 & 1 & 10 & 233 & 12 \\ 7 & 5 & 1 & 11 & 1249 & 34 \\ 8 & 14 & 2 & 12 & 7595 & 130 \\ 9 & 50 & 5 & & & \end{array}$

The authors are indebted to the University of California (Berkeley) Computer Center for the use of its machines.

University of California

Berkeley, California 94533

1. Robert Bowen, "The generation of minimal triangle graphs," Math. Comp.,

2. D. W. Grace, "Computer search for non-isomorphic convex polyhedra," Ph.D. Dissertation, Stanford University, Stanford, Calif., 1965. 